\title{
Four electrons interacting pairwise in the limit of infinitesimal confining potentials: especially the quintet spin state.
}

\author{
Ali Akbari, ${ }^{1,2,3}$ Claudio Amovilli, ${ }^{4}$ Norman H. March, ${ }^{1,5,6}$ and Angel Rubio ${ }^{1,2,3,7}$ \\ ${ }^{1}$ Departamento de Fisica de Materiales, Facultad de Químicas, UPV/EHU, Apdo 1072, 20080 San Sebastián, Spain \\ ${ }^{2}$ Centro de Fisica de Materiales, CSIC UPV/EHU, Paseo M.de Lardizabal 5, 20018 San Sebastián, Spain \\ ${ }^{3}$ European Theoretical Spectroscopy Facility (ETSF), Centro Joxe \\ Mari Korta, Avenida de Tolosa, 72, 20018 San Sebastián, Spain \\ ${ }^{4}$ Department of Chemistry and Industrial Chemistry, University of Pisa, Pisa, Italy \\ ${ }^{5}$ Department of Physics, University of Antwerp, Antwerp, Belgium \\ ${ }^{6}$ Oxford University, Oxford, England \\ ${ }^{7}$ Donostia International Physics Center, Paseo M.de Lardizabal 4, 20018 San Sebastián, Spain
}

\begin{abstract}
In very recent work, Amovilli and March have considered the Hookean atom with four electrons. In particular, they demonstrated the cross-over from a triplet $P_{g}$ ground state at large harmonic force constant $k$ to a quintet $S_{u}$ configuration for weak confinement, by means primarily of diffusion quantum Monte Carlo simulation. Here, we focus on such a quintet state for again 4 spin half fermions but now interacting via a harmonic pair force $-K r_{i j}$. The spatial quintet $\Psi$ is then available , with its eigenvalue. Progress on calculating low-order spinless density matrices is recorded. Finally, this prompts us to examine the Amovilli-March results when extrapolated to small $k \neq 0$. We display as $k \rightarrow 0^{+}$approximate results for the four-electron Hookean atom model, including fitted analytic forms for kinetic and Coulomb energy as a function of $k$ as $k \rightarrow 0^{+}$.
\end{abstract}

Keywords: Density matrix, Moshinsky atom, Hookean atom

A very recent study by Amovilli and March [1] of the Hookean atom with four Coulombically repelling electrons has had recourse to diffusion quantum Monte Carlo (DQMC) simulations. The authors demonstrated thereby, for a harmonic confining external potential $V_{\text {ext }}(r)$ given by

$$
V_{\text {ext }}(r)=\frac{1}{2} k r^{2}
$$

that, as $k$ is varied from strong to weak values, there is a cross-over from a triplet ground-state ${ }^{3} P_{g}$ to a quintet configuration ${ }^{5} S_{u}$, the ${ }^{3} P_{g}$ being lowest in energy for large $k$.

Here, we first complement the above DQMC study for the four-electron Hookean atom by re-opening the Moshinsky model [2], but now for four electrons. However, to make analytical progress, we first put $k=0^{+}$in Eq.(1) and consider the four electrons moving under the influence of a central pairwise interaction $V\left(r_{i j}\right)$ given, following [2] for two electrons by

$$
V\left(r_{i j}\right)=\frac{1}{2} K r_{i j}^{2}
$$

Prompted by the comments on [1] made above, we shall restrict ourselves to the quintet, $S=2$, state, however. It is then fortunate that the wave function, denoted below by $\Psi^{(2 S+1)}=\Psi^{(5)}$, is known, its calculation going back, at very least, to Bruch [3].

The form of $\Psi^{(5)}$ is then given by (see Eqs.(12), (1) and (36) of [3]) as a product of a spatial wave function $B$ and a spin function. Since we shall work with the reduced spatial density matrices $\Gamma_{n}$ of Löwdin [4] below, we need not concern ourselves with the spin function. But $B$ is known and hence we can write the spatial four-electron $\Psi^{(5)}\left(\mathbf{r}_{1}, \mathbf{r}_{2}, \mathbf{r}_{3}, \mathbf{r}_{4}\right)$ explicitly as (Eq.(36) of [3]), in unnormalized form:

$$
\Psi^{(5)}\left(\mathbf{r}_{1}, \mathbf{r}_{2}, \mathbf{r}_{3}, \mathbf{r}_{4}\right)=\mathbf{u} \cdot(\mathbf{v} \times \mathbf{w}) \exp Q .
$$

Bruch gives the RHS of Eq.(3), explicitly defining $\mathbf{u}$ to $Q$ as follows:

$$
\begin{gathered}
\mathbf{u}=\frac{1}{2}\left(\mathbf{r}_{1}+\mathbf{r}_{2}\right)-\frac{1}{2}\left(\mathbf{r}_{3}+\mathbf{r}_{4}\right), \\
\mathbf{v}=\mathbf{r}_{1}-\mathbf{r}_{2}, \mathbf{w}=\mathbf{r}_{3}-\mathbf{r}_{4}
\end{gathered}
$$

and

$$
Q=-\frac{m \omega_{I}}{4 \hbar}\left(2 u^{2}+v^{2}+w^{2}\right) .
$$


Here $\omega_{I}$ is related to $K$ introduced in Eq.(2) by

$$
\omega_{I}^{2}=\frac{K}{m}
$$

Defining, as in [3], an energy unit $\epsilon$ by

$$
\epsilon=\hbar\left(\frac{K}{m}\right)^{(1 / 2)}
$$

the quintet wave function Eq.(3) corresponds to total energy $15 \epsilon$ [3].

It is evident that with total Hamiltonian $\hat{H}$, which separates into a centre-of-mass kinetic energy term and three decoupled oscillators, we can then write the 'local energy' equation as

$$
15 \epsilon=\frac{\hat{H} \Psi^{(5)}\left(\mathbf{r}_{1}, \mathbf{r}_{2}, \mathbf{r}_{3}, \mathbf{r}_{4}\right)}{\Psi^{(5)}\left(\mathbf{r}_{1}, \mathbf{r}_{2}, \mathbf{r}_{3}, \mathbf{r}_{4}\right)}
$$

Following, for example, Dawson and March [5], we can reduce the above Eq.(9) by straightforward manipulation into an equation of such local energy form relating solely the reduced density matrices $\Gamma^{(3)}, \Gamma^{(2)}$ and $\gamma\left(\mathbf{r}_{1}^{\prime}, \mathbf{r}_{1}\right)$. The integration of $\Gamma^{(3)}$ to find the exact correlated $\Gamma^{(2)}$ involves fairly extensive but straightforward manipulation. It leads to the closed form of $\Gamma^{(2)}$ as

$$
\begin{aligned}
\Gamma^{(2)}\left(\mathbf{r}_{1}^{\prime}, \mathbf{r}_{2}^{\prime} ; \mathbf{r}_{1}, \mathbf{r}_{2}\right)= & \frac{\sqrt{2} \pi^{3} \hbar^{4}}{2 m^{4} \omega^{4}}\left\{\left(\frac{4 \hbar}{m \omega}-\left|\mathbf{R}-\mathbf{R}^{\prime}\right|^{2}\right) \mathbf{r} \cdot \mathbf{r}^{\prime}+(\mathbf{R} \cdot \mathbf{r})\left(\mathbf{R} \cdot \mathbf{r}^{\prime}\right)+\left(\mathbf{R}^{\prime} \cdot \mathbf{r}^{\prime}\right)\left(\mathbf{R}^{\prime} \cdot \mathbf{r}\right)\right. \\
& \left.-\left(\mathbf{R}^{\prime} \cdot \mathbf{r}\right)\left(\mathbf{R} \cdot \mathbf{r}^{\prime}\right)-(\mathbf{R} \cdot \mathbf{r})\left(\mathbf{R}^{\prime} \cdot \mathbf{r}^{\prime}\right)\right\} \mathbf{e}^{-\frac{m \omega}{4 \hbar}\left(\left|\mathbf{R}-\mathbf{R}^{\prime}\right|^{2}+\mathbf{r}^{2}+\mathbf{r}^{\prime 2}\right)}
\end{aligned}
$$

where $\mathbf{r}$ and $\mathbf{R}$ are defined (the primed quantities are defined in the same way but with primes instead) by

$$
\mathbf{R}=\frac{\mathbf{r}_{1}+\mathbf{r}_{2}}{2}, \quad \mathbf{r}=\mathbf{r}_{1}-\mathbf{r}_{2}
$$

The diagonal element of Eq.(10) is the very important pair density $P(r)=\Gamma^{(2)}\left(\mathbf{r}_{1}, \mathbf{r}_{2} ; \mathbf{r}_{1}, \mathbf{r}_{2}\right)$ where $r=\left|\mathbf{r}_{1}-\mathbf{r}_{2}\right|$. $P(r)$ is then of the form

$$
P(r)=\frac{2 \sqrt{2} \pi^{3} \hbar^{5}}{m^{5} \omega^{5}} r^{2} e^{-\frac{m \omega}{2 \hbar} r^{2}} .
$$

But equally important is the $1 \mathrm{DM} \gamma\left(\mathbf{r}_{1}^{\prime}, \mathbf{r}_{1}\right)$, which is given by

$$
\gamma\left(\mathbf{r}_{1}^{\prime}, \mathbf{r}_{1}\right)=\int \Gamma^{(2)}\left(\mathbf{r}_{1}^{\prime}, \mathbf{r}_{2} ; \mathbf{r}_{1}, \mathbf{r}_{2}\right) d \mathbf{r}_{2}
$$

with $\Gamma^{(2)}$ in Eq.(10) after putting $\mathbf{r}_{2}^{\prime}=\mathbf{r}_{2}$. The result has the form

$$
\gamma\left(\mathbf{r}_{1}^{\prime}, \mathbf{r}_{1}\right)=24 \pi^{\frac{9}{2}}\left(\frac{\hbar}{m \omega}\right)^{\frac{11}{2}}\left\{\frac{\hbar^{2}}{m^{2} \omega^{2}}-\frac{\hbar}{8 m \omega} r^{2}\right\} e^{-\frac{3 m \omega}{16 \hbar} r^{2}}
$$

where here $r$ denotes $\left|\mathbf{r}_{1}-\mathbf{r}_{1}^{\prime}\right|$. To proceed to relate the pair density $P(r)$ to $\gamma\left(\mathbf{r}_{1}^{\prime}, \mathbf{r}_{1}\right)=\gamma\left(\left|\mathbf{r}_{1}-\mathbf{r}_{1}^{\prime}\right|\right)$, we note next that from Eq.(12)

$$
\frac{m^{5} \omega^{5} P(r)}{2 \sqrt{2} \pi^{3} \hbar^{5} r^{2}}=e^{-\frac{m \omega}{2 \hbar} r^{2}}
$$

Taking logarithms of Eq.(15) yields immediately that

$$
-\frac{m \omega}{2 \hbar} r^{2}=\ln \frac{m^{5} \omega^{5} P(r)}{2 \sqrt{2} \pi^{3} \hbar^{5} r^{2}}
$$

This can be substituted into Eq.(14) with the result that

$$
\gamma\left(\mathbf{r}_{1}^{\prime}, \mathbf{r}_{1}\right)=24 \pi^{\frac{9}{2}}\left(\frac{\hbar}{m \omega}\right)^{\frac{11}{2}}\left\{\frac{\hbar^{2}}{m^{2} \omega^{2}}+\frac{1}{16} \ln \frac{m^{5} \omega^{5} P(r)}{2 \sqrt{2} \pi^{3} \hbar^{5} r^{2}}\right\}\left(\frac{m^{5} \omega^{5} P(r)}{2 \sqrt{2} \pi^{3} \hbar^{5} r^{2}}\right)^{\frac{3}{8}}
$$


It remains to eliminate $\omega$ from Eq.(17) to find what from the theorems of DFT could be a universal relationship in systems with translational invariance. Returning to Eq.(14) and taking its diagonal form yields

$$
n(r)=\gamma\left(\mathbf{r}_{1}, \mathbf{r}_{1}\right)=\gamma(0)=24 \pi^{\frac{9}{2}}\left(\frac{\hbar}{m \omega}\right)^{\frac{15}{2}} .
$$

Hence we find

$$
\frac{\hbar}{m \omega}=\left(\frac{\gamma(0)}{24 \pi^{\frac{9}{2}}}\right)^{\frac{2}{15}}
$$

and therefore we relate through Eq.(17) and Eq.(19) the $\gamma$ and $\Gamma^{(2)}$ via the diagonal (constant) element of $\gamma(r)$ given in Eq.(19).

To conclude this letter, we return to the Hookean atom with four electrons in [1] in the limit of weak harmonic confinement $k \rightarrow 0$. Then in Fig. 1 we have plotted the result for $T$ versus $\omega=\left(\frac{k}{m}\right)^{1 / 2}$, where $T$ is the total kinetic energy. We have used both the definitions of kinetic energy tabulated in Table II by Amovilli and March [1]. The dashed curve shown is motivated by the study of Cioslowski and Grzebielucha [6]. These authors found the exact limiting behavior of the total energy $E$ for four electrons trapped in harmonic external potential as $k \rightarrow 0^{+}$. The leading terms from their result read

$$
E=\frac{9}{2^{2 / 3}} \omega^{2 / 3}+\frac{1}{4}(6+4 \sqrt{3}+3 \sqrt{6}) \omega+\ldots .
$$

The curve of Fig.1 is then the plotted term

$$
T=-0.22 \omega^{2 / 3}+3.54 \omega
$$

Turning to Fig.2, this depicts the Coulomb potential $U_{c}$, data being again taken from Table II of [1], and used in the relation $U_{c}=(2 / 3)(U-T)$ obtained from the virial theorem, $U$ being the total potential energy including the external potential contribution. With the same functional form, the analytical fit is

$$
U_{C}=4.00 \omega^{2 / 3}-1.02 \omega
$$

In summary, the achievements of this letter are (a) concerned with the four-electron Moshinsky model and (b) the weak trapping limit of the analogous Hookean atom to (a). For this model (a), we can get some exact analytical relations for low-order spinless density matrices. Finally, we stress the key results in Eq.(17) and Eq.(19), which in the translationally invariant four-electron model relate the one and two DMs; a long-term aim of many-body theory.

\section{ACKNOWLEDGMENT}

N. H. March (NHM)wishes to thank Professor P. M. Echenique for most generous hospitality and much stimulation at DIPC where NHM's contribution to the present study was carried out. The continuing affiliation of NHM with the University of Antwerp (UA) was made possible by Professors D. Lamoen and C. Van Alsenoy through BOF-NOI(UA). We acknowledge financial support from Spanish MEC (FIS2007-65702-C02-01), ACI-Promociona (ACI2009-1036), Grupos Consolidados UPV/EHU del Gobierno Vasco (IT-319-07), Spanish Research Council (CSIC) and the European Research Council Advanced Grant DYNamo (ERC-2010-AdG-Proposal No. 267374).

[1] C. Amovilli and N. H. March, Phys. Rev. A 83 (2011), p. 044502.

[2] M. Moshinsky, Am. J. Phys. 36 (1998), p. 52.

[3] L. W. Bruch, J. Chem. Phys. 72 (1980), p. 5511.

[4] P. O. Löwdin, Phys. Rev. 97 (1955), p. 1474.

[5] K. A. Dawson and N. H. March, J. Chem. Phys. 81 (1984), p. 5850.

[6] J. Cioslowski and E. Grzebielucha, Phys. Rev. A 77 (2008), p. 032508. 


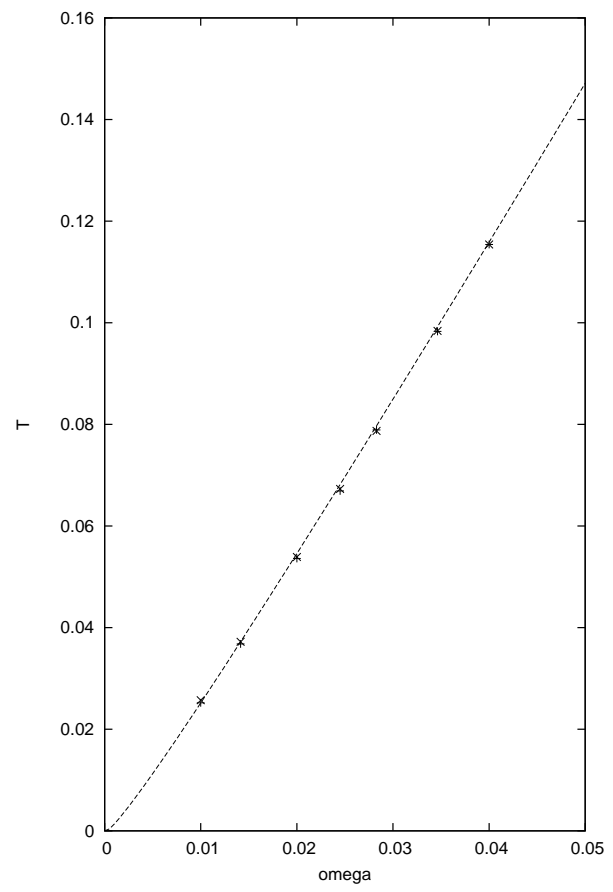

FIG. 1: Total kinetic energy $T$ of Hookean atom with four Coulombically repelling electrons as function of confining force constant $k$ in Eq.(1), where $\omega^{2}$ is proportional to $k$. Note that both forms of kinetic energy obtained from the quantum Monte Carlo calculation in [1] have been used. The dashed curve shows the fitted analytic curve displayed in Eq.(21).

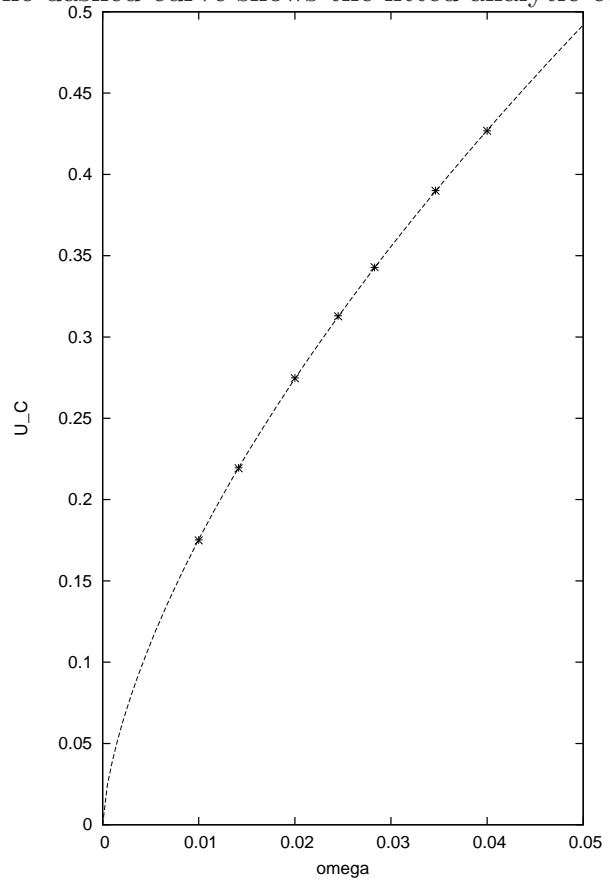

FIG. 2: Shows plot of Coulomb potential energy $U_{c}$ versus $\omega$ for Hookean atom with four electrons Crosses shows results of Amovilli and March [1] using diffusion QMC calculations. Dashed curve is given by Eq.(22) of text, motivated by the analytic work for small $\omega$ in [6]. 